\title{
Analyzing Optimal Setting Of Reference Point Group Mobility Model Using DSR Protocol In MANETS
}

\author{
Nasser Ali Husieen \\ Department of Computer Science, College of Education \\ Wasit University \\ Kut, Iraq \\ Email: dr.naseer.alquraishi@gmail.com
}

Mohammad M. Rasheed

Information Technology Directorate

Ministry of Science and Technology

Baghdad, Iraq

Email: mohmadmhr@yahoo.com

\begin{abstract}
Mobile Ad-hoc Network (MANET) is a self-configuring wireless network. The nodes can configure themselves to be in any arbitrary topology. It is essential that mobility models used in simulating different scenarios must emulate closely the real scenario in order to assess the MANET performance as accurately as possible. In this paper, the authors have studied the effect of different maximum pause times and maximum node speeds on different performance metrics in order to arrive at optimal settings for these two attributes under the Reference Point Group Mobility model for the DSR protocol. In addition, this work is part of an ongoing research on link failures in DSR protocol. Thus, the performance of the DSR protocol under the Reference Point Group Mobility (RPGM) model in terms of different pause times, node speeds, number of nodes and number of source connections were evaluated. The simulation results show that the maximum pause time and the maximum speed have direct impacts on the performance parameters such as packet delivery ratio, routing overhead, average end-to-end delay, normalized routing load and packet drop under the Reference Point Group Mobility model.
\end{abstract}

Keywords-MANET, Mobility Models, Routing Protocols, DSR Protocol, RPGM

\section{INTRODUCTION}

Mobile ad hoc network is a collection of wireless nodes that allows devices to communicate with each other without help of an existing infrastructure [1]. A MANET is a self configuring and a self-organizing such that it can create an arbitrary topology temporarily for short while in which mobile nodes work as both routers and end nodes. MANET based applications range from military applications connecting soldiers in a battlefield, social networks for communication during emergencies such as natural disasters to personal area networks. New applications such as telemedicine, weather reporting and dissemination and disaster information dissemination are emerging in the recent times exploiting the new developments and the advantages of MANETs compared to the traditional infrastructure based networks. The above mentioned developments will increase the size and reach of MANETs to thousands of nodes which is hitherto limited to few nodes in both military and civil application domains.

One of the main challenges in setting up and managing a dynamic mobile ad hoc network is routing. Routing protocols detect the optimum path between the source node and destination node in a complex network of nodes and deliver the data packets between those nodes in an efficient manner. In mobile ad hoc networks, high mobility, limited computing capability and low bandwidth associated with nodes make routing of data one of the most challenging tasks. Researchers have proposed different routing protocols to address these issues. These protocols can be categorized into two main groups. They are namely on demand or reactive protocols and periodic or proactive protocols. Protocols such as Dynamic Source Routing (DSR) [2], Ad-hoc On-Demand Distance Vector Routing ( AODV) [3] and Temporally ordered routing algorithm (TORA) [4] coming under the reactive protocols establish the route only prior to sending the packets and maintain the route only when it is needed. The proactive protocols such as Destination-Sequenced Distance Vector (DSDV) [5] on other hand periodically exchange routing information to maintain the routes continuously. In general, reactive protocols have been shown to outperform the proactive protocols due to their reduced overhead and ability to react quickly when routes change [6-9]. This motivated us to investigate more into on demand protocols. One of the most important and difficult tasks in simulating mobile ad hoc networks is the definition of a mobility model to represent real world scenarios. In the real world, mobile networks encounter various situations including intense mobility creating heavy 
uncertainties especially in disaster situations. Hence taking measurements under real world conditions is an almost impossibility. The alternative to real world measurements is to simulate the different situations using a computer software represent the real world situation as closely as possible and take the measurements. Presently in the mobile ad hoc environments, the following mobility models have been used; Random Way Point, Manhattan Grid, Gauss Markov mobility model and Reference Point Group Mobility (RPGM) model [10]. In this paper, we use the RPGM model implemented in the Network Simulator 2 (NS2) version 2.34 under Linux to study the effect of pause time and node speed on the DSR protocol. Different scenarios were simulated and the performance of the network was critically evaluated with special reference to these parameters. The main objective of the analysis was to determine the optimal settings for these two parameters.

The rest of the paper is organized as follows: Section 1 provides an introduction to the study. Sections 2 and 3 discuss RPGM model in details and related work respectively. Details of the simulation environment setup and results are presented in Sections 4 and 5. Finally Sections 6 concludes the paper along with suggestions for future work.

\section{DyNAMIC SOURCE ROUTING PROTOCOL}

Dynamic Source Routing (DSR) protocol is a simple reactive routing protocol developed by Johnson at the Carnegie Mellon University in 1996. The key feature of the DSR protocol is based on the source routing where full or partial route is specified by the sender [11]. In mobile ad hoc networks, the source routing technique provides several advantages, including flexibility, simplicity and correctness [12-14]. When a node wants to send data packets to another node, the intermediate nodes forwarding the packets towards the detonation need not maintain up to date routing information as the data packet will contain information on how to forward the packet. DSR protocol is made up of two mechanisms namely, Route Discovery and Route Maintenance. DSR uses route cache to store the routes to other nodes. The main advantage of using route cache is it speeds up route discovery and reduces the propagation of route request packets. DSR can also provide interconnection between wireless devices with multiple network interfaces. This is vital in strategic communications as nodes in the military require communicating with different devices at different ranges

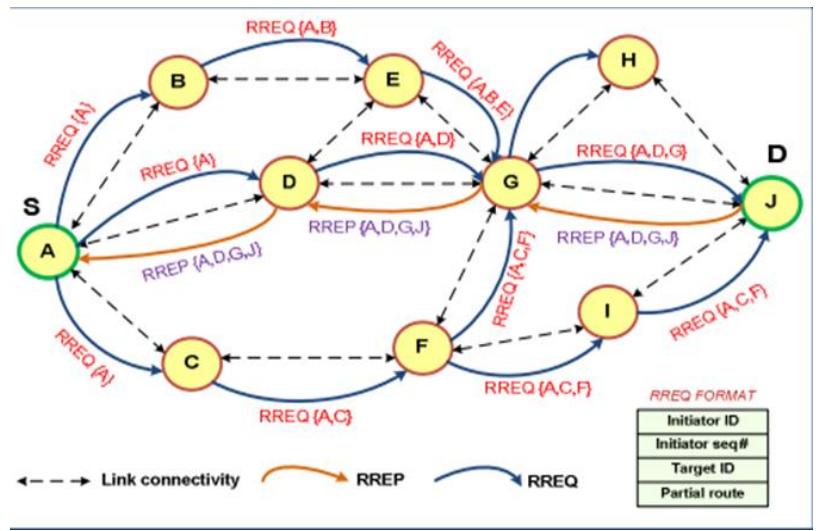

Fig.1. Route Discovery Mechanism
Fig. 2 shows the route maintenance mechanism of DSR protocol. In this mechanism, DSR used two types of packets: ACKs packet is used for correction and verification operation of the routes, and second type of packets is called Route Error Packet (RRER). RRER is generated when there is link failure occurs between intermediate nodes and next hop due to battery usage, hard medium contention, and the node mobility that is leading to loss the packets as shows in the figure below when the link fails between the intermediate node I and destination node D.

Fig. 1 shows the route discovery mechanisms. In this figure, when a source node (A) wants to send data packets to the destination node $(\mathrm{J})$. First, it checks if there is a route to the destination in the route cache, route request will be discarded. Otherwise, source node (A) broadcasts Route Request Packet (RREQ) to the neighbour nodes $(\mathrm{B}, \mathrm{C}, \mathrm{D})$ within wireless transmission range of a node $(\mathrm{A})$. The route request identifies the destination node $\mathrm{J}$ to which a route in needed. If the neighbour nodes (B, C, D) are not the target, locally these neighbour nodes rebroadcast RREQ to the next hop after adding its own address to a list of nodes in the route cache. Each RREQ packet contains source address, destination address, request ID, and route record. When a route request packet arrives at the target node $(\mathrm{J})$, the destination node $(\mathrm{J})$ returns a Route Reply Packet (RREP) along with the reverse of a recorded path to the source node (A), which is $(A, D, G$, $J)$. When a source node (A) receives more than one RREP for a given destination, it selects the first route that receives RREQ in order to reduce the time for route discovery packet. DSR protocol is made up of two mechanisms namely, Route Discovery and Route Maintenance. DSR uses route cache to store the routes to other nodes. The main advantage of using route cache is it speeds up route discovery and reduces the propagation of route request packets.

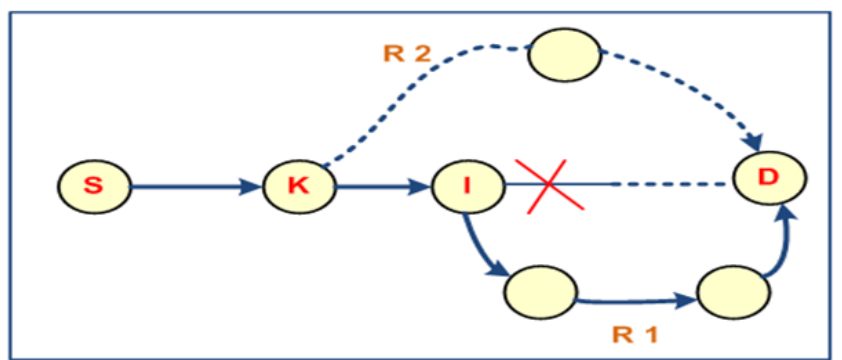

Fig.2. Route Maintenance Mechanism 


\section{REFERENCE POINT GROUP MOBILITY MODEL}

The Reference Point Group Mobility (RPGM) model was proposed by Hong [15]. In this model, all nodes work as a group and the nodes of the group moves as a single entity to achieve different tasks. Each group has the logical centre called the group leader. The path of the group in its entirety is represented by the locus of the centre. Each node in the group has its own reference point for communicating with other nodes. The reference point of the node follows the group movement; the real location of the node can be determined by its reference point plus a random motion vector that denotes its stability from the reference point. Reference point Group mobility is adapted for several applications such as a battlefield situation where a number of soldiers move together in a group, disaster recovery and convention scenarios. According to Hong's report, the RPGM outperforms Random Way Point model in case of link failures due to the inherent characteristic of spatial dependency between nodes. The RPGM model incurs fewer link breakages and achieves better performance for various routing protocols compared to Random Way Point model. The functions of the group leader and group members are as follows:

\section{A. Group Leader}

$V_{\text {group }}^{t}$ It provides the general motion movement of the whole group. Each member of this group moves away from this group motion. The motion vector $V_{\text {group }}^{t}$ can be arbitrarily selected or carefully designed based on several predefined paths.

\section{B. Group members}

The group members' movement is heavily affected by its group leader's movement. Each mobile node is assigned with a reference point that follows the group movement. With respect to this predefined reference point, every mobile node might be arbitrarily located in the neighborhood. Formally, the motion vector of the group members $\mathrm{i}$, at the time $\mathrm{t}, \mathrm{V}_{\mathrm{i}}^{\mathrm{t}}$ can be defined as:

$$
\mathrm{V}_{\mathrm{i}}^{\mathrm{t}}=\mathrm{V}_{\text {group }}^{\mathrm{t}}+\mathrm{RM}_{\mathrm{i}}^{\mathrm{t}}
$$

Where $R M_{i}^{t}$ is the random motion vector representing the deviation of the group member $i$ from its reference point. The vector $R M_{i}^{t}$ is free identically distributed random procedure whose duration is uniformly distributed in the interval $\left[0, r_{\text {max }}\right]$, where $r_{\max }$ is maximum acceptable distance and whose path is uniformly distributed in the interval $[0,2 \pi]$.

Fig. 3 illustrates the Reference Point Group Mobility model with the group leader represented in green and the members represented in red and yellow respectively. $V_{\text {group }}^{\mathrm{t}}$ is the motion vector of the group leader and the whole group.

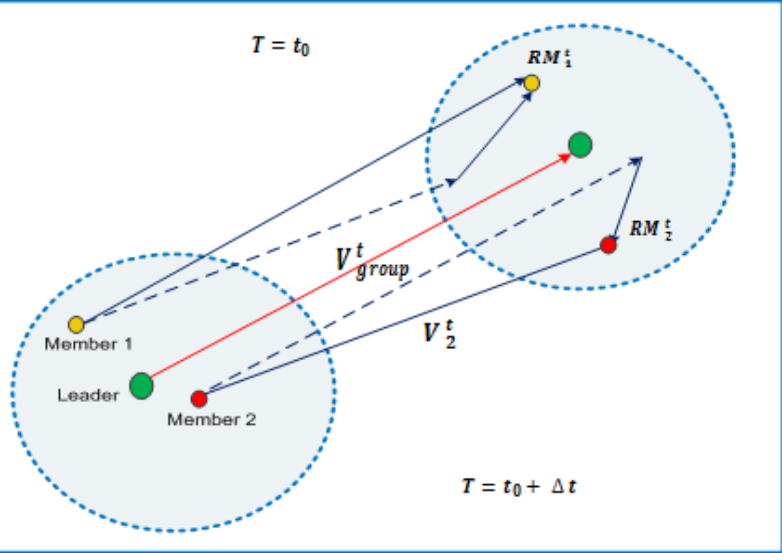

Fig.3. Node Movement in RPGM Model

With appropriate selection of the predefined paths for the group leader and other parameters, the RPGM model can emulate a variety of mobility behaviours. The RPGM model is thus able to represent various mobility scenarios, such as:

- In-Place Mobility Model: In this model, the whole field is divided into adjacent regions. Each region is totally occupied by a single group. An example of this model is battlefield communication.

- Overlap Mobility Model: In this model, different groups with different tasks move on the same field in an overlapping style. An example of this model is Disaster relief.

- Convention Mobility Model: In this model, the area is divided into few regions and several groups are permitted to move between the regions. An example of this model is a conference.

In RPGM model, the vector $\mathrm{RM}_{\mathrm{i}}$ indirectly determines how far the group members deviate from their leader. The movement can be characterized as follows:

$\left|V_{\text {member }}(t)\right|=\left|V_{\text {leader }}(t)\right|+$ random $0 * S D R *$
max_speed

$\theta_{\text {member }}(t)=\theta_{\text {leader }}(t)+$ random $0 * A D R *$ max_angle

Where $0<$ SDR, ADR $<1$,

SDR is the Speed Deviation Ratio and

ADR is the Angle Deviation Ratio

SDR and ADR are used to control the deviation of the velocity in terms of both magnitude and direction of group members from that of the leader. Different mobility scenarios can be generated by adjusting these two parameters

\section{RELATED WORK}

Different mobility models have different impacts on the performance of mobile ad hoc routing protocols [16]. Different protocols have different metrics that capture interesting mobility characteristics like spatial and temporal dependence and geographic restrictions. Hong et al [15] introduced a mobility model called the Mobility Vector (MV) model and compared the performance of different routing protocols such as DSR, AODV and FSR against other mobility models including Random Way Point, RPGM and Random Walk. Packet delivery ratio and link Up/Down were measured using simulation varying the average speed and transmission range respectively. Hence it is important to select the right mobility model to represent the real scenario under 
consideration. Geetha and Gopinath compared the performance of two on demand routing protocols, namely AODV and DSR under different mobility models such as Random Way Point and Reference Point Group Mobility in order to characterize the two routing protocols under the different mobility models [17]. Simulation parameters computed included fixed pause time at $25 \mathrm{~s}$, five low traffic source and speeds up to $20 \mathrm{~m} / \mathrm{s}$. In this study, the authors concentrate on different pause times and maximum speed with increased number of source connection along with increased node density in order to find the optimal setting of pause time and node speed for the DSR protocol in the Reference Point Group Mobility.

Kioumourtzis evaluated the performance of OLSR, AODV and DSR three reactive protocols under Manhattan-Grid, and Reference Point Group mobility models [18]. The main objective of his work was to compare the performance of each protocol under the two models in order to understand the limitations of the protocols. The simulations were carried out under different conditions. The number of nodes was varied up to 90 , the number of connections was increased up to 40 , the packet rate was also increased and the nodes' speeds of movement were fixed at 5, 10, 15 and $20 \mathrm{~m} / \mathrm{s}$ along with a pause time of $5 \mathrm{~s}$. However, this paper presents the performance of the DSR protocol with increased node density, increased CBR traffic and various nodes' mobility velocities up to $80 \mathrm{~m} / \mathrm{s}$ with different pause times up to $40 \mathrm{~s}$ in order to select the optimal setting for the DSR protocol under Reference Point Group Mobility model.

Agrawal, Tiwari and Vyas have evaluated the AODV protocol under four different mobility models, namely the Manhattan-Grid, Markov-Grid, Random Way Point and Reference Point Group Mobility model [19]. Their objective was to select a suitable model for AODV protocol. The metrics used by them were packet delivery ratio and delay. Their simulation result shows that the AODV performs well with RPGM model in terms of packet delivery ratio and end to end delay. The simulation parameters used were fixed pause time of $10 \mathrm{~s}$, different velocities of $5,10,15,20,25 \mathrm{~m} / \mathrm{s}$ and increased network load of 4, 8, 12 and 16 packets per second. The work presented by this paper focuses on velocities up to $80 \mathrm{~m} / \mathrm{s}$ with different pause times up to 40 with the objective of selecting the average optimal setting for these two parameters.

\section{SIMULATION ENVIRONMENT}

The Network Simulator 2 (NS2) version 2.34 installed on Centos Linux operation system was used as the simulation tool in this work. In NS2 the node movement has to be defined in a OTCL script or be imported from an external file. In this project, the mobility scenarios were created using the Bonn Motion version 1.4 a Java software tool specifically designed for this purpose [20]. Bonn Motion was developed by the Communication Systems Group at the Institute of Computer Science 4 of the University of Bonn, Germany. This tool can generate the most common mobility models such as Random Way Point, Gauss-Markov and Manhattan-Grid models. In this project, the Reference Point Group mobility (RPGM) model has been used as a movement model and the cbrgen.tcl generator tool which is located under the directory indeputils/cmu-scen-gen has been used to generate random source traffic. The traffic pattern used is CBR. Fig. 4 shows the simulation methodology adopted in this work. Each scenario was simulated for 200 seconds within a simulated rectangular geographical area of $1000 \mathrm{~m} \times 500 \mathrm{~m}$. Tables 1 and 2 lists the rest of simulation parameters. 


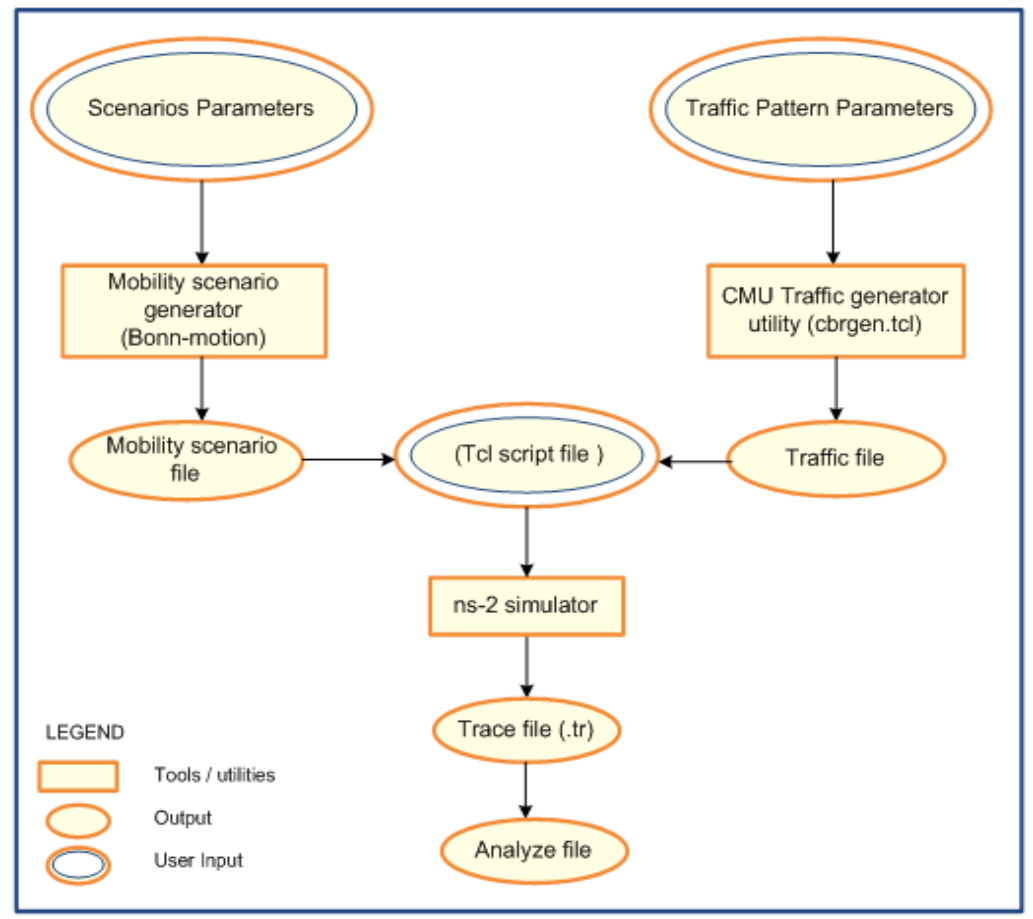

Fig 4. Simulation Methodology

TABLE 1: Simulation Parameters

\begin{tabular}{|l|l|}
\hline Parameters & Value \\
\hline Simulation Time & $200 \mathrm{~s}$ \\
\hline Number of nodes & $10,20,40,80$ \\
\hline Number of connections & $4,8,30,40$ \\
\hline Maximum Pause Time & $5,10,20,30,40$ \\
\hline Simulation Area & $1000 \times 500 \mathrm{~m}$ \\
\hline Minimum node speed & $0 \mathrm{~m} / \mathrm{s}$ \\
\hline Maximum node speed & $20,40,60,80 \mathrm{~m} / \mathrm{s}$ \\
\hline Mobility Model & RPGM \\
\hline Routing Protocol & DSR \\
\hline Traffic Type & $\begin{array}{l}\text { Constant Bit Rate } \\
(\mathrm{CBR})\end{array}$ \\
\hline Packet size & 512 bytes \\
\hline Distribution of nodes & 5 groups \\
\hline Probability of group change & 0.05 \\
\hline $\begin{array}{l}\text { Maximum distance to group } \\
\text { centre }\end{array}$ & $100 \mathrm{~m}$ \\
\hline
\end{tabular}

\begin{tabular}{|l|l|}
\hline Standard deviation & 2.0 \\
\hline MAC Type & 802.11 \\
\hline
\end{tabular}

According to the data listed in Table 1, there are eight main scenarios comprising four main scenarios of different speeds and another four main scenarios of different pause times. In the case of first main scenarios, for each speed was tested under four sub-scenarios by changing different metrics at a time making the total number of scenarios under this category to be 16 . Under each pause time, five sub-scenarios were created using different additional parameters making the total number of scenarios under this category to be 20 . Thus, the total number of scenarios tested in the experiment is 36 . Since the performance of ad hoc routing protocols is sensitive to the movement pattern, scenario files were generated with 50 different movement patterns representing 10 movement

$$
\begin{aligned}
& . / b m-f \text { scenatiol }-b \text { RPGM }-n 80-d 200-x 1000-y \\
& 500-h 20.0-l 0.0-p 20.0-a 5.0-c 0.05-r 100-s \\
& 2.0
\end{aligned}
$$

patterns per pause time. 
Table 2 lists the parameters used in generating the RPGM mobility model in Bonn Motion.

The following command will generate the Reference Point Group Mobility scenario.

TABLE 2: RPGM Parameters in Bonn Motion

\begin{tabular}{|l|l|}
\hline Parameters & Explanation \\
\hline$-\mathrm{n}$ & Number of mobile nodes \\
\hline$-\mathrm{d}$ & Simulation duration time \\
\hline$-\mathrm{x}$ & Simulation area width \\
\hline$-\mathrm{y}$ & Simulation area height \\
\hline$-\mathrm{c}$ & Group change Probability \\
\hline$-\mathrm{l}$ & Highest velocity \\
\hline$-\mathrm{h}$ & Pause time \\
\hline$-\mathrm{p}$ & $\begin{array}{l}\text { Average number of nodes } \\
\text { per group }\end{array}$ \\
\hline$-\mathrm{a}$ & Maximum distance to group \\
\hline$-\mathrm{r}$ &
\end{tabular}

\begin{tabular}{|l|l|}
\hline & centre \\
\hline$-s$ & $\begin{array}{l}\text { Group size standard } \\
\text { deviation }\end{array}$ \\
\hline
\end{tabular}

After the generation of scenario1, the following command should be typed to transform the scenario1 into a file that can be read by ns-2. Fig. 5 shows the creation of clusters as shown in the NAM console at completion of the simulation.

\section{.$/ b m$ NSFile - f scenariol}

\section{SIMULATION RESUlTS}

Four different scenarios were considered with different pause times. Each scenario contained five sub scenarios along with four different scenarios for different node speeds containing four sub scenarios each. The simulation for five times for each scenario and the average of the results were computed. The packets delivery ratio, average end to end delay, routing overhead, normalized routing load and packet drop were used as metrics in evaluating performance.

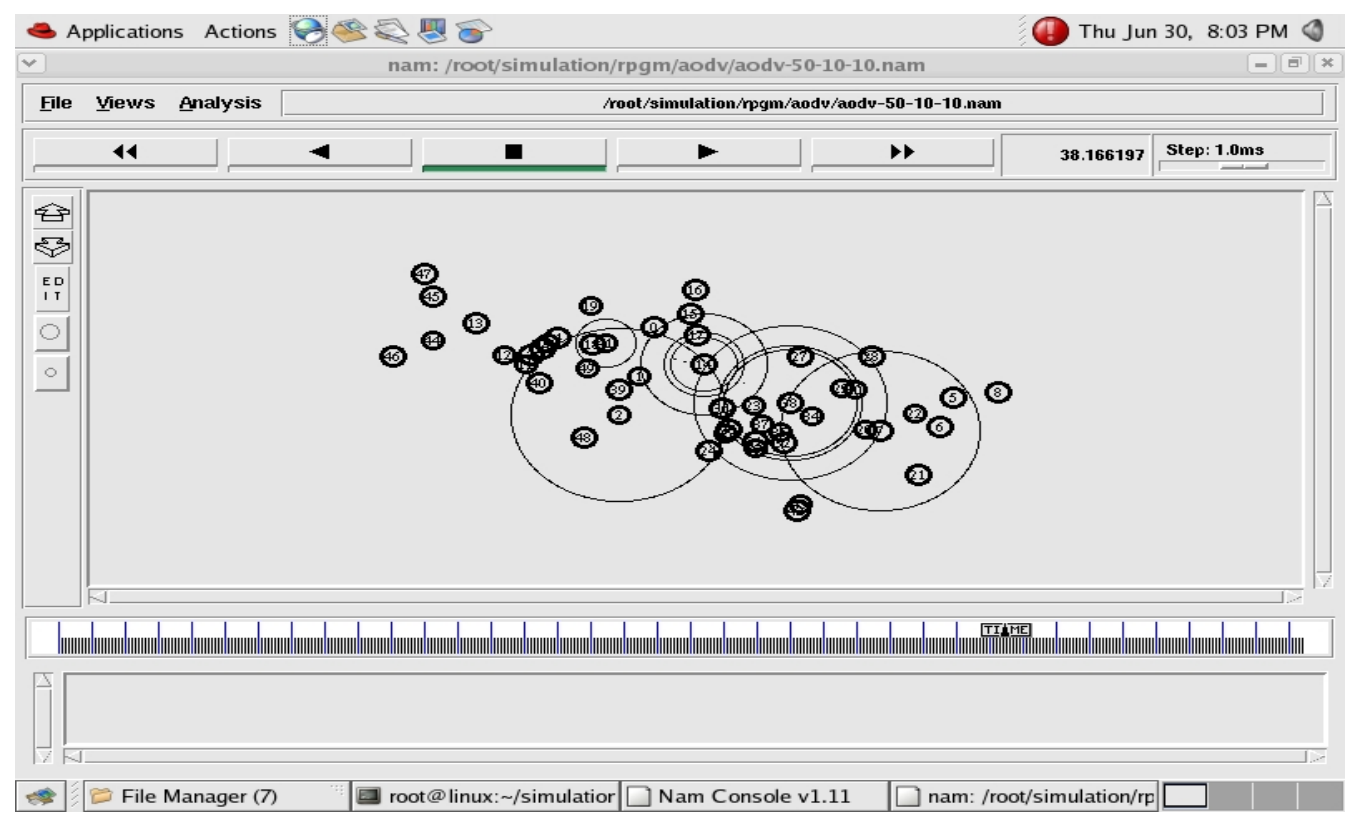

Fig. 5. Creation of Clusters with the RPGM model 


\section{A. Packet Delivery Ratio}

Packet Delivery Ratio (PDR) is defined as the fraction of the number packets received at the destination to the number of packets originated by the source application. PDR describes the loss rate seen by the transport layer protocols. This will affect the overall network throughput.

The PDR as a percentage is given by Formula (3).

$$
\left[P D R \%=\frac{\sum_{1}^{n} \text { DATArecv }}{\sum_{1}^{n} \text { DATAsent }} \times 100\right]
$$

Fig. 6. (A) shows the PDR for different pause times. The investigation was carried at pause times 5, 10, 20, 30 and $40 \mathrm{~s}$ and the PDR at these pause times were measured in order to select the best pause time. From Fig. 6 (A), it can be seen that the DSR protocol performs well when the pause time is $20 \mathrm{~s}$ under all the four scenarios. The results show that the highest PDR with the maximum number of packets sent and received at the pause time $20 \mathrm{~s}$, is $100.00 \%, 100.00 \%, 99.35 \%$ and $99.96 \%$. After selecting the pause time to be fixed at $20 \mathrm{~s}$, different maximum speed of $20,40,60$ and $80 \mathrm{~m} / \mathrm{s}$ were investigated in order to select the optimal node speed in the RPGM model.

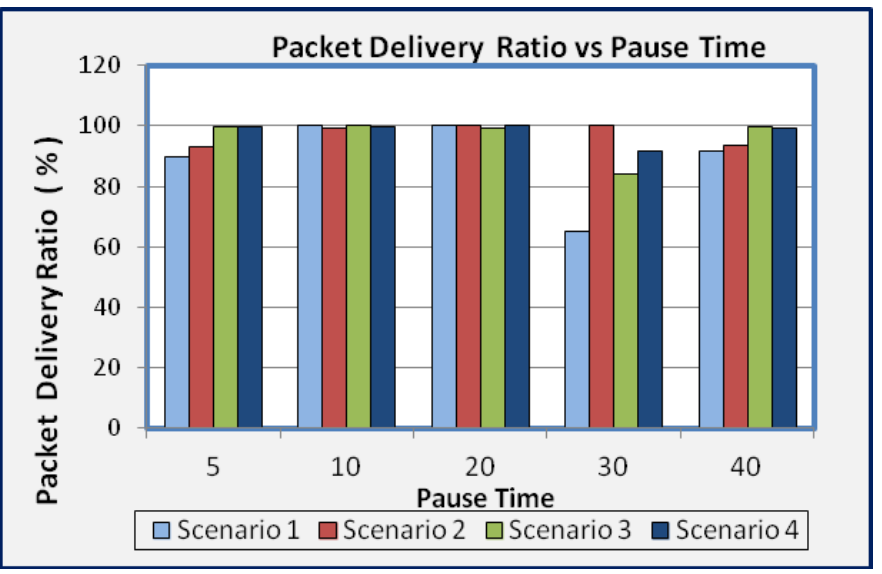

Fig. 6 (A). PDF vs. Pause time

Figure 6(B) shows the effect of node speed on the DSR protocol. The results show that the highest PDR is achieved when maximum speed is $20 \mathrm{~m} / \mathrm{s}$ or $72 \mathrm{~km} / \mathrm{h}$. The PDR at 20 $\mathrm{m} / \mathrm{s}$ were $100.00 \%, 99.96 \%, 99.97 \%$ and $99.88 \%$ respectively. Whenever the node speed was increased, the PDR dropped due to the reason that the group leader and the members were moving very fast during the packet delivery process. This causes packets to drop leading to reduced PDR. It was also observed that when a mobile node moves fast and the pause time is small; the topology is likely to be in a highly dynamic condition. Hence, the optimal setting for node speed and the pause time are $20 \mathrm{~m} / \mathrm{s}$ and $20 \mathrm{~s}$ respectively.

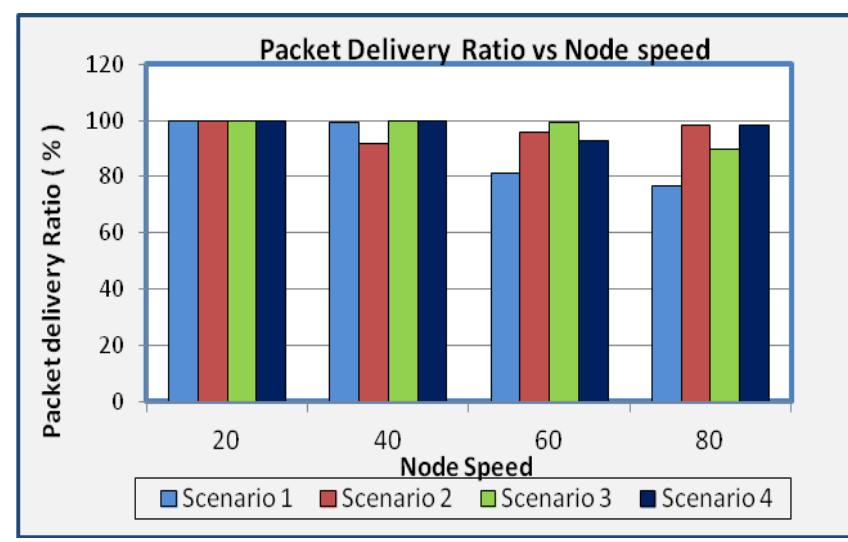

Fig. 6 (B). PDF vs. Node speed

\section{B. Average End-to-End Delay}

Average end-to-end delay is defined as the average time taken by a packet to reach the destination from the source. The average end-to-end delay includes all the types of delays such as transmission delay, propagation delay, processing delay and interface queuing delay. The average end-to-end delay between two nodes is computed using Formula (4).

$$
\left[\text { Avg_Delay }=\frac{\sum_{1}^{n} \text { DATAsent-DATArecv }}{\sum_{1}^{n} \text { DATArecv }}\right]
$$

The delay is affected by higher CBR packet generation rate at the source as well. When packets are generated at high rates, the buffer at the source becomes full resulting in longer queuing delays at the source node. Figures 8(A) and (B) show the effect of maximum pause time and speed on average endto-end delay respectively. Fig. 7(A) shows that the average end to end delay is reduced when the pause time is $20 \mathrm{~s}$ in the 2nd and 4th scenarios. The average end-to-end delays were $30.56 \mathrm{~s}$ and $15.82 \mathrm{~s}$ respectively. The average end to end delays in the $1 \mathrm{st}$ and $3 \mathrm{rd}$ cases at $10 \mathrm{~s}$ pause time were $6.62 \mathrm{~s}$ and $11.66 \mathrm{~s}$ respectively. The reason for this discrepancy is the increased time consumption for route discovery at $20 \mathrm{~s}$ pause time due to buffer overflow in the 1 st and 3rd scenarios.

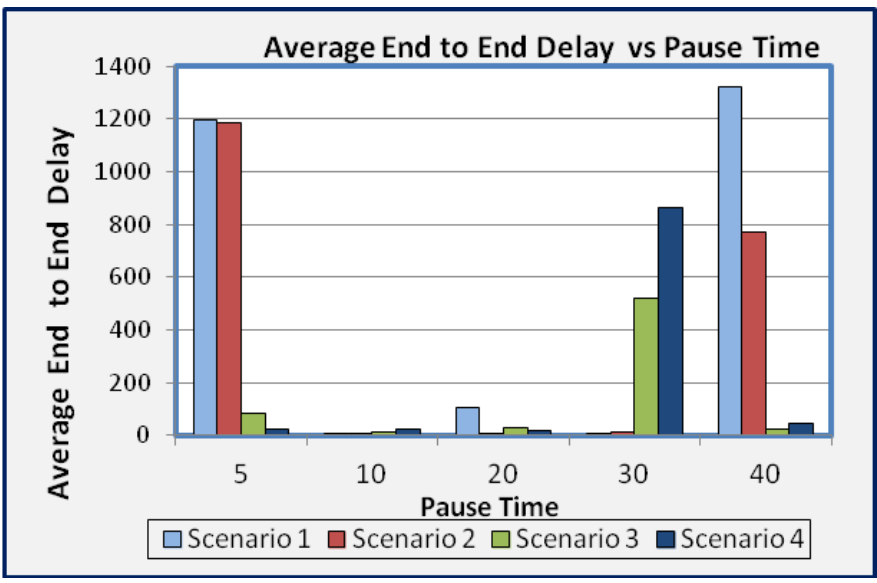

Fig 7(A). Delay vs. Pause Time

Fig. 7(B) shows the effect of different maximum speeds on the average of end-to-end delay. From Fig. 7(B) it can be seen that 
the average end-to-end delay is decreased when maximum speed is $20 \mathrm{~m} / \mathrm{s}$. An increase in maximum speed also results in an increase in the average end-to-end delay. This is due to the reason that when a node moves fast, it causes link failures leading to new route discovery processes. New route discovery processes would create additional delays increasing the overall end-to-end delay. Hence it can be concluded that the optimum settings for the maximum pause time and the speed are $20 \mathrm{~s}$ and $20 \mathrm{~m} / \mathrm{s}$ respectively.

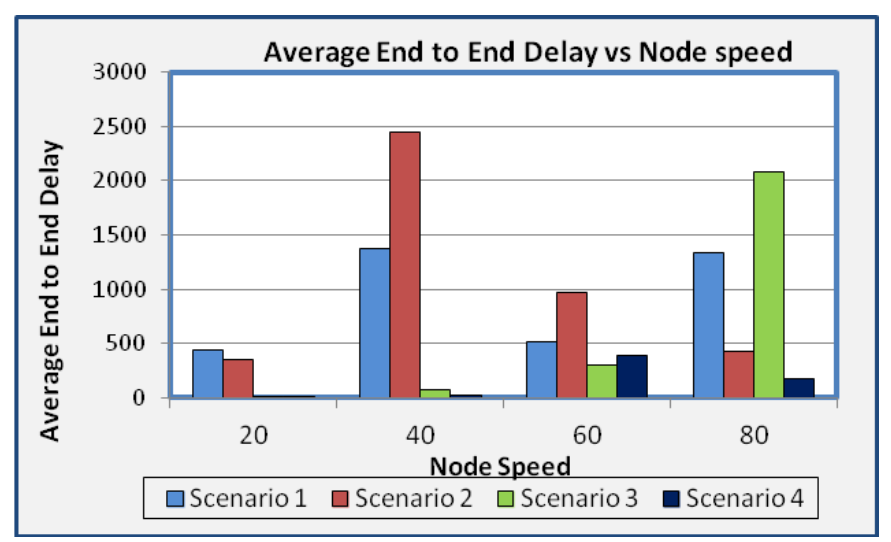

Fig. 7 (B). Delay vs. Node Speed

\section{Routing Overhead}

Overhead is an important issue as higher overheads reduce the overall network resources utilization. Hence it is important to reduce the overhead in a network as much as possible. Fig. 8 (A) and (B) show the routing overhead for different pause times. From Fig. 9(A), it can be seen that different pause times affect the routing overhead under all the four scenarios. Fig. 8 (A) shows that the overhead is the minimum when maximum pause time is $20 \mathrm{~s}$, except for the 3rd scenario with total number of nodes to be equal to 40 with 30 source connections and the maximum pause time to be $10 \mathrm{~s}$. The $3 \mathrm{rd}$ scenario has the optimum setup at the maximum pause time equal to $10 \mathrm{~s}$ due to the lower number of packets transmitted. In addition, DSR protocol uses a route cache to reduce the number of route discovery processes during the establishment and transmission of packets.

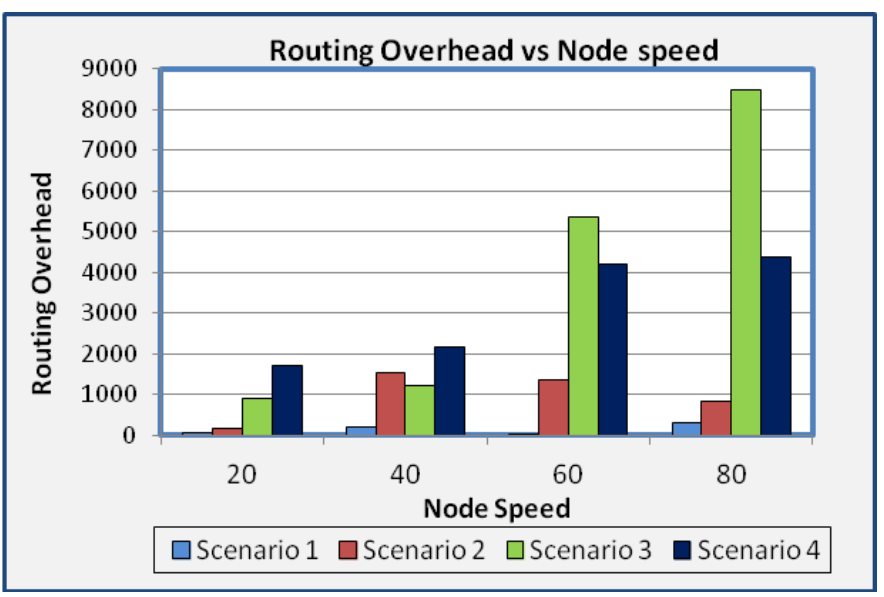

Fig. 8 (A). Overhead vs. Pause time
Fig. 8(B) shows that different maximum speeds affect the routing overhead. It can be seen that the routing overhead is the minimum at the maximum speed is at $20 \mathrm{~m} / \mathrm{s}$ in all scenarios. Whenever the maximum speed of a node is increased, the overhead is also increased. Hence, it can be concluded that the average optimal settings for maximum pause time and speed are $20 \mathrm{~s}$ and $20 \mathrm{~m} / \mathrm{s}$ for minimizing the routing overhead.

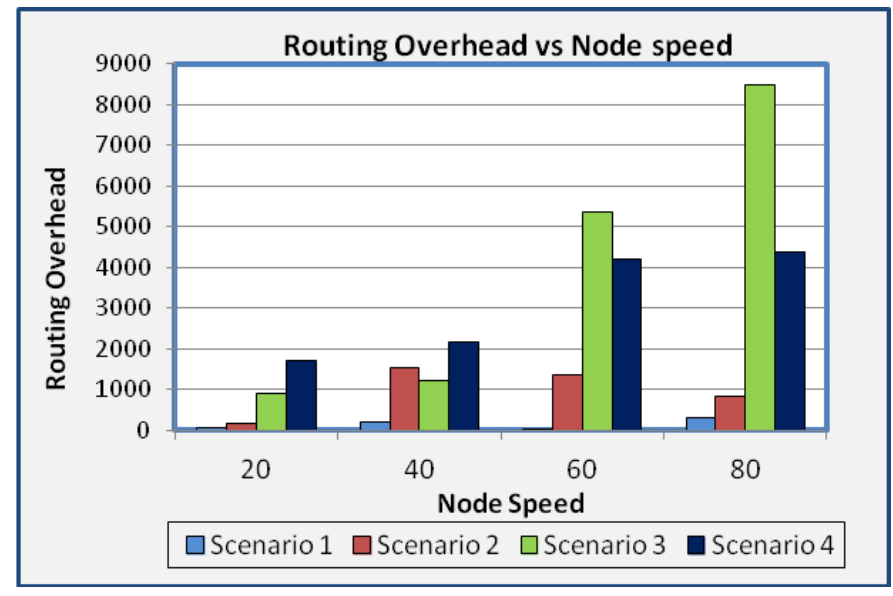

Fig. 8 (B). Overhead vs. Node speed

\section{Normalized Routing Load (NRL)}

The Normalized Routing Load (NRL) is an estimate of how efficient a routing protocol is. The number of routing packets sent per data packet is an indication of how well the protocol maintains the routing information updated. The higher the normalized routing load, the higher the overhead of routing packets is and consequently the lower the efficiency of the protocol. Fig. 9(A) and (B) show the results for the NRL against different maximum pause times and maximum node speed respectively. Fig. 9(A) shows that the DSR protocol performs best when maximum pause time is $20 \mathrm{~s}$ except for the 3rd scenario, where the pause time is $10 \mathrm{~s}$. This is due to the reason that the NRL is directly proportional to the overhead and the packets sent. As shown in Fig. 10(A), the NRL is reduced when the maximum pause time is $10 \mathrm{~s}$.

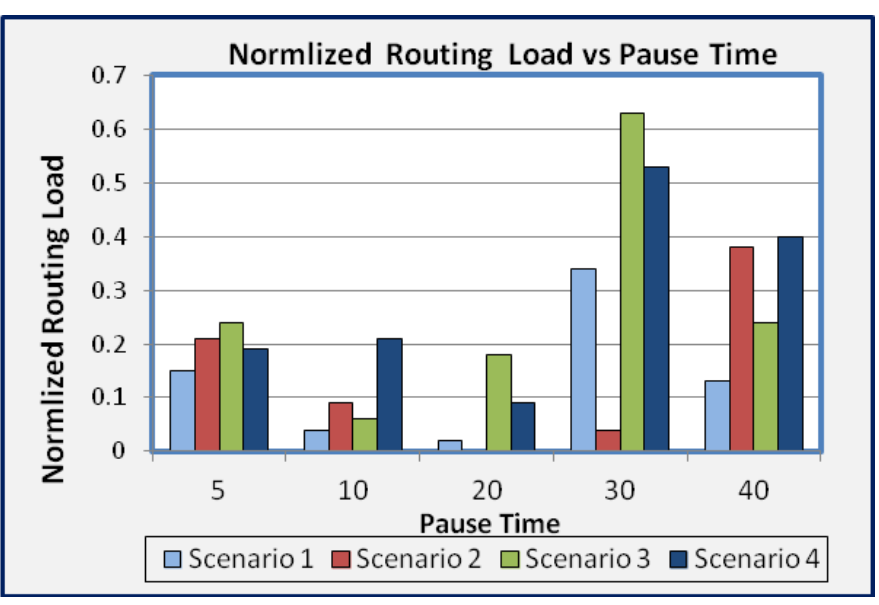

Fig. 9 (A). NRL vs. Pause time 
Fig. 9(B) shows the effect of the different maximum node speeds on the NRL. Fig. 9(B) shows that the DSR protocol performs best when maximum node speed is $20 \mathrm{~m} / \mathrm{s}$ resulting in the lowest NRL value. Whenever the maximum node speed is increased, the NRL is also increased leading to lower efficiency. Hence, it can be concluded that the optimal setting for the maximum pause time and the maximum node speed are $20 \mathrm{~s}$ and $20 \mathrm{~m} / \mathrm{s}$ respectively for efficient NRLs.

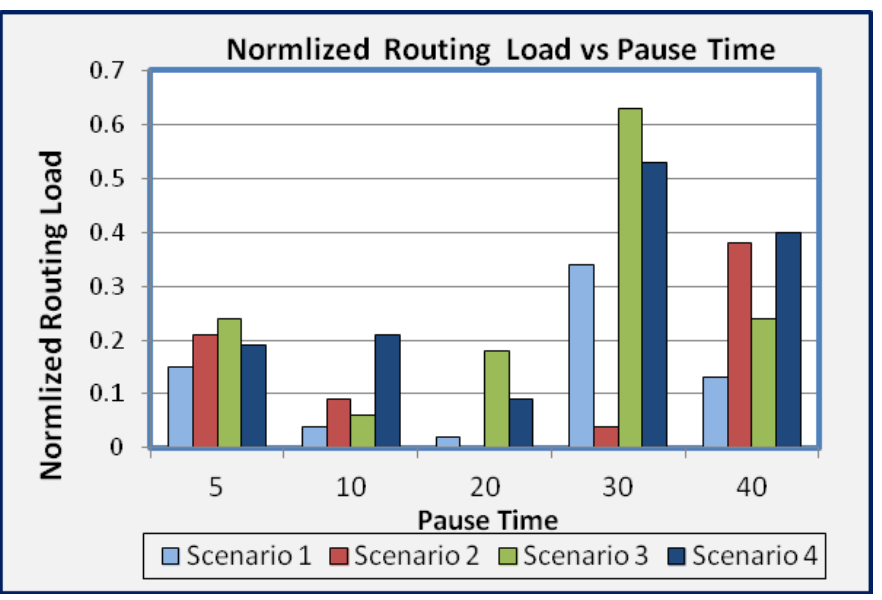

Fig. 9( B). NRL vs. Node speed

\section{CONCLUSIONS}

In this paper, the authors have presented the work of evaluating the performance of the DSR protocol under Reference Point Group Mobility model with respect to the effects of the maximum pause time and maximum speed under different scenarios. The simulation parameters included 36 different scenarios of which 20 scenarios are for different maximum pause times and 16 scenarios are for different maximum node speeds. Simulation results show that the maximum pause time and speed have a direct impact on the performance of the DSR protocol. The Reference Point Group Mobility model has two constraints. One is that the movement of nodes should follow that of the group leader and the other one is that each group leader has the group movement limited to certain speed limits. The simulation results show that when the mobility is high, the possibility of route caches becoming stale is also high resulting in frequent link failures. When a route discovery is initiated, the large number of replies received in response is associated with high MAC overhead causing increased interference with data traffic. Hence, the cache staleness and high MAC overhead together result in significant degradation of performance in DSR in high mobility scenarios. Furthermore, simulation results show that there are four main factors that can affect the DSR protocol. They are namely, the maximum node speed, the maximum pause time, the number of connections, and the number of nodes. From the simulation results, it was observed that the optimal settings for the maximum pause time and the maximum node speed are $20 \mathrm{~s}$ and $20 \mathrm{~m} / \mathrm{s}$ respectively.

\section{REFERENSES}

[1] J. Jain, M. Fatima, and R. Gupta, "Overview Challenges of Routing Protocol and Mac Layer in Mobile Ad-hoc Network," Journal of Theoretical and Applied Information Technology vol. 8, pp. 6-12, 2009.

[2] C. E. Perkins and E. M. Royer, "Ad-Hoc on-Demand Distance Vector Routing," in The Second IEEE Workshop on Mobile Computing Systems and Applications, 1999, pp. 90-100.

[3] M. S. Corson and A. Ephremides, "A Distributed Routing Algorithm for Mobile Wireless Networks," Journal of Wireless Networks, vol. 1 , pp. 61-81, 1995.

[4] C. E. Perkins and P. Bhagwat, "Highly Dynamic DestinationSequenced Distance-Vector Routing (DSDV) for Mobile Computers," in SIGCOMM '94 Conference on Communications Architectures, Protocols and Applications, 1994, pp. 234-244.

[5] J. Broch, D. A. Maltz, D. B. Johnson, Y. Hu, and J. G. Jetcheva, "A Performance Comparison of Multi-Hop Wireless Ad Hoc Network Routing Protocols," in Fourth Annual ACM/IEEE International Conference on Mobile Computing and Networking 1998, pp. 85-97.

[6] P. Johansson, T. Larsson, N. Hedman, B. Mielczarek, and M. Degermark, "Scenario-based Performance Analysis of Routing Protocols for Mobile Ad-hoc Networks," Fifth Annual ACM/IEEE International Conference on Mobile Computing and Networking, pp. 195-206, 1999.

[7] D. A. Maltz, J. Broch, J. Jetcheva, and D. B. Johnson, "The Effects of On-Demand Behaviour in Routing Protocols for Multi-Hop Wireless Ad Hoc Networks," IEEE Journal on Selected Areas in Communications, Special Issue on "Wireless Ad Hoc Networks, vol 1439-1453, pp. 1439-1453, 1999.

[8] S. R. Das, C. E. Perkins, and E. M.Royer, "Performance Comparison of Two On-demand Routing Protocols for Ad Hoc Networks," in Nineteenth Annual Joint Conference of the IEEE Computer and Communications Societies 2000, pp. 3-12.

[9] P. Prabhakaran and R. Sankar, "Impact of Realistic Mobility Models on Wireless Networks Performance," in Wireless and Mobile Computing, Networking and Communications, 2006, pp. 329 - 334

[10] D. B. Johnson, "Routing in Ad Hoc Networks of Mobile Hosts," in IEEE Workshop on Mobile Computing Systems and Applications, 1994, pp. 158-163.

[11] D. B. Johnson and D. A. Maltz, "Dynamic Source Routing in Ad Hoc Wireless Networks," in Mobile Computing, ed, 1996, pp. 153-181.

[12] D. B. Johnson, D. A. Maltz, and Y. Hu. (2004). Dynamic Source Routing Protocol for Mobile Ad-hoc Networks (DSR). Available: http://www.ietf.org/internet-drafts/draft-ietf-manet-dsr-10.txt

[13] D. B. Johnson, D. A. Maltz, Y.-C. Hu, and J. G. Jetcheva. (2001). The Dynamic Source Routing Protocol for Mobile Ad Hoc Networks, Available: Internet- Draft, draft-ietf-manet-dsr-05.tx

[14] D. B. Johnson, D. A. Maltz, and J. Broch, "The Dynamic Source Routing Protocol for Multi-hop Wireless Ad Hoc Networks," in Ad Hoc Networking, ed, 2001, pp. 139-172.

[15] X. Hong, M. Gerla, G. Pei, and C. C. Chiang, "A Group Mobility Model for Ad Hoc Wireless Networks," in 2nd ACM International Workshop on Modelling, Analysis and Simulation of Wireless and Mobile Systems, 1999, pp. 53-60.

[16] F. Bai, N. Sadagopan, and A. Helmy, "Important: a Framework to Systematically Analyze the Impact of Mobility on Performance of Routing Protocols for Ad Hoc Networks," in 22nd Annual Joint Conference on the IEEE Computer and Communications Societies USA, 2003, pp. 825-835.

[17] G. Jayakumar and G. Gopinath, "Performance Comparison of Two Ondemand Routing Protocols for Ad-hoc Networks based on Random 
ICIT 2015 The $7^{\text {th }}$ International Conference on Information Technology

doi:10.15849/icit.2015.0032 C ICIT 2015 (http://icit.zuj.edu.jo/ICIT15)

Waypoint Mobility Model," Journal of Applied Sciences American, vol. 6, pp. 659-664, 2008.

[18] G. Kioumourtzis, "Simulation and Evaluation of Routing Protocols for Mobile Ad Hoc Networks," MSc. Thesis, Naval Postgraduate School, California, 2005

[19] C. P. Agrawal, M. K Tiwari, and O. P. Vyas, "Evaluation of AODV Protocol for Varying Mobility Models of MANET for Ubiquitous Computing," in Proceeding of the Third International Conference on Convergence and Hybrid Information Technology, 2005.
[20]

Bonn

Motion

Mobility www.informatik.unibonnde/IV/BonnMotion.
Generator 